\title{
Correlation Between Clinical Oral Dryness Score and Salivary Flow Rates in Active and Passive Smokers
}

\author{
Seeme Nigar, Sobia Hassan, Sidra Fahim, Ahmed Bin Khalid, Faisal Salim
}

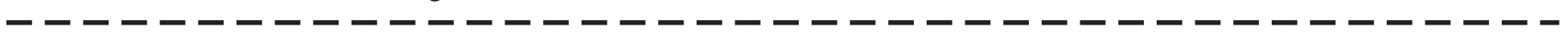

ABSTRACT

Objective: To evaluate the mean clinical oral dryness score (CODs) and salivary flow rate (SFR) in subjects exposed to active and passive smoking. The secondary aim was to determine the correlation between CODs and SFR and potential factors associated with CODs and SFR in our subjects.

Study Design and Setting: The clinical observational study was conducted at the Outpatient department of oral diagnosis at Altamash Institute of Dental Medicine, Karachi from Jan 2019-Jul 2019.

Methodology: A total of 217 participants of ages 15 - 80years coming for routine dental checkup of either gender were included. A thorough oral examination was performed for all the subjects. A non-stimulated saliva sample was collected from the oral cavity of subjects in a graduated container. The salivary flow rate was noted in $\mathrm{ml} / \mathrm{min}$ for $5 \mathrm{minutes}$. Assessment of oral dryness/hypo-salivation was examined through CODs (clinical oral dryness score). The data were analyzed using SPSS software version 23.

Results: The mean COD score and SFR were calculated as 1 and $0.42 \mathrm{ml} / 5 \mathrm{~min}$ respectively. The Pearson's correlation between COD score and SFR level was calculated as -0.281 (negative correlation) such as the SFR significantly decreased when the COD score increased $(\mathrm{p}<0.05)$. The age, gender, smoking status, betel quid and areca nut consumption showed statistically significant difference in mean COD score and SFR levels $(\mathrm{p}<0.05)$.

Conclusion: Salivary flow rate was significantly decreased with increase in COD score thus having an implication on oral dryness feeling in these participants.

Keywords: Areca nut, Betel quid, Mouth dryness, Hypo-salivation, Smoking.

\section{INTRODUCTION:}

The oral tissues are continuously bathed with saliva in healthy individuals. The continuous flow of saliva helps to maintain oral hemostasis and helps in defense against microorganisms. ${ }^{1-3}$ The salivary flow rate shows great variation from $0.3-0.65 \mathrm{ml} / \mathrm{min}$ level to $1.5-6 \mathrm{ml} / \mathrm{min}$ when stimulated by chewing or by mild strength citric acid. ${ }^{4}$ The salivary glands have secretions which plays vital role in the protection of mucosa from feeling of dryness. The feeling

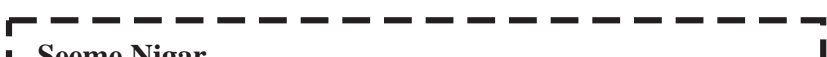

Seeme Nigar

MSc Trainee, Department of Oral Pathology

I Altamash Institute of Dental Medicine,

I Defense, Karachi

I Sobia Hassan

I Associate Professor, Department of General Pathology

Altamash Institute of Dental Medicine,

I Defense, Karachi

I Sidra Fahim

I Senior Lecturer, Department of Oral Medicine/Periodontology

I Altamash Institute of Dental Medicine,

Defense, Karachi

I Email: sidra.raf@gmail.com

I Ahmed Bin Khalid

I MSc Trainee, Department of Periodontology

I Altamash Institute of Dental Medicine,

Defense, Karachi

Faisal Salim

Senior Dental Surgeon, Health Department

I Government of Baluchistan, Quetta

Received: 22-Oct-2019

I Accepted: 07-Apr-2020

L Accepted. 07-Apr-2020 of dry mouth is a subjective and complex condition and affects approximately $46 \%$ of the population. ${ }^{5}$ Clinically, mouth dryness may differ from a small decrease in salivary flow with temporary inconvenience to severe deterioration of oral health and concomitant psychosomatic indisposition. The hypofunction of saliva has mainly been associated with decrease in salivary flow rate (SFR) and estimated as below $3 \mathrm{iL} / \mathrm{cm}^{3} / \mathrm{min}^{1,2,6}$ Hence, the most appropriate technique for the diagnosis salivary gland disorder is to measure SFR and it can be used as easily available, safe and non-invasive diagnostic modality to diagnose different systematic and oral conditions. ${ }^{7,8}$

Clinical oral dryness score (CODS) is a new tool designed to assess oral dryness by clinical and visual inspection of the oral cavity based on several signs of oral dryness such as the presence of frothy saliva, the dryness of the oral mucosa and stickiness of the dental mirror to the tongue or the buccal fold. CODS closely determines both the unstimulated salivary flow and the thickness of mucin layer over the epithelium (mucosal wetness) suggesting a physiological basis to the feeling of dryness. ${ }^{9}$ Osailan et al. also suggested CODS as a reliable tool for the estimation of hyposalivation severity. ${ }^{3}$

Hence, literature has shown varying results such as weak to no correlation between mouth dryness and SFR. ${ }^{5,10-12}$ In Pakistan, there is lack of baseline data, and international data isn't applicable in our population due to variation in genetic and personal habits. Therefore, the aim of current study was to evaluate the mean CODS and SFR among 
patients exposed to active $\&$ passive smoking. The secondary aim was to determine the degree of correlation between CODS and SFR in these patients. The potential factors associated with changes in SFR and CODS were also determined in this study.

\section{METHODOLOGY:}

The clinical observational study was conducted at the department of oral pathology at Altamash Institute of Dental Medicine, Karachi from January 2019-July 2019. The sample size was estimated using WHO sample size calculator, by taking statistics for mean salivary flow rate as $1.93 \pm 0.65^{13}$, keeping margin of error as $8.65 \%$ and confidence level as $95 \%$. The non-probability consecutive sampling technique was employed which gave a sample size of 217 patients in total. All the patients of 15-80 years coming for routine dental checkup of either gender were included in the study. Patients who were taking systemic medications such as betablockers or steroids, patients with severe head or neck injury, undergoing radiotherapy, wearing dentures, patients with learning disability or pregnant women were excluded from the study.

Approval from ethical review committee was obtained prior to the commencement of study. All the participants were informed about the nature of the study and written informed consent was taken. A pre-designed proforma was used to collect the demographic information, along with the data regarding smoking habit of each individual (frequency and duration). An individual who had smoked $>100$ cigarettes (including hand-rolled cigarettes, cigarillos, cigars) in their lifetime and had smoked at least one cigarette in the past month or 28 days were labelled as active smokers. The data regarding the consumption of betel quid (pan) and areca nut (chalia) was also obtained. An individual who had consumed betel quid or areca nut more than 100 times in their lifetime and had consumed at least five times in the past month or 28 days were labelled as consumers. A thorough oral examination was performed for all the subjects. Each subject was advised not to eat, drink, smoke or perform any oral hygiene methods for a minimum of 60 minutes before and during the collection of the saliva sample. The subject was seated upon the dental chair. A non-stimulated saliva sample was collected from the oral cavity of each subject who were asked to drool in a graduated cup every 1 minute for 5 minutes, in a ventilated and well illuminated room. The saliva collection was performed within the working hours of the OPD at Altamash Institute of Dental Medicine, Karachi. SFR was measured in $\mathrm{ml} / 5 \mathrm{~min}$. Assessment of oral dryness/ hypo-salivation was examined through CODS (clinical oral dryness score) ${ }^{14}$. The data were analyzed using SPSS computer software version 22. Quantitative variables such as age, COD score and salivary flow rate (SFR) were reported as mean and standard deviation. Qualitative variables like gender, ethnicity, smoking status and consumption of betel quid and areca nut were reported as frequency and percentage.
Effect modifiers like age, gender, ethnicity, smoking status, consumption of betel quid and areca nut were addressed through stratification. Post-stratification independent t-test was applied. P-value of $<0.05$ was taken as statistically significant.

\section{RESULTS:}

In this study, a total of 217 participants attending for routine dental checkups were evaluated. The mean age of the study participants was reported as $32.86 \pm 6.30$ years ranging from 22- 55 years. Out of 217 participants, 145 were males $(66.8 \%)$ whereas 72 were females (33.2\%). More than half of the participants were Urdu speaking (58.5\%) and $19.8 \%$ spoke in Sindhi. About 24.9\% of the participants were active smokers and average number of cigarettes smoked by them per day was reported as 5.09 ranging from 1-11 cigarettes per day. Out of 217 participants, $16.6 \%$ were betel quid and areca nut consumers. (Table 1)

Figure 1 shows the average COD score to be 1 within the range of 0-4. Whereas, Figure 2 shows that the mean SFR was $0.42 \mathrm{ml} / 5 \mathrm{~min}$ ranging from $0.2-1 \mathrm{ml} / 5 \mathrm{~min}$. The Pearson's correlation between COD score and SFR level was calculated as -0.281 (negative correlation). It was inferred from our results that CODS was a significant predictor of SFR, as the value of SFR significantly decreased with an increase in the COD score $(\mathrm{p}<0.05)$.

Stratification of mean COD score and SFR ml/5min was done with respect to potential effect modifiers. The age, gender, smokers, betel quid and areca nut consumers showed statistically significant difference in mean COD score and SFR level $(\mathrm{p}<0.05)$. (Table 2)

\section{DISCUSSION:}

In last few decades,multiple researches have reported different aspects of mouth dryness and salivary flow rate. Yet, there is a big gap in the evidence for relation between salivary flow rate and oral dryness. In the present study, the mean CODS was estimated as $1 \pm 1.12$ in patients coming for routine dental check up and mild dryness (COD score 1-3) was present in majority of them. A study conducted by et al. also demonstrated similar results in healthy controls i.e. mean CODS of $1.0 \pm 0.9$ whereas SFR value in our study were estimated at $0.42 \pm 0.10 \mathrm{ml} / 5 \mathrm{~min} .{ }^{3}$ Hijjaw $\mathrm{O}$ et al. in their research found the mean stimulated whole saliva flow rate as $0.46( \pm 0.44) \mathrm{mL} / 5 \mathrm{~min}$ which is slightly higher than that seen in present study. ${ }^{10}$ In the present study, a negative weak correlation was observed between CODS and SFR $(r=-0.281)$. Hence the results suggest that flow of saliva significantly decreased when the COD score increased $(\mathrm{p}<0.05)$. In line with our findings, Correia et al. found moderate negative correlation between CODS and SFR ( $\mathrm{r}=-$ $0.515, \mathrm{p}<0.05$ ). Patients with no oral dryness (CODs 0-1) had high rate of unsimulated SFR $>0.5 \mathrm{ml} / 5 \mathrm{~min}$ whereas patients with moderate dryness had decreased rate of unsimulated SFR $>0.1 \mathrm{ml} / 5 \mathrm{~min}(\mathrm{p}<0.01) .{ }^{15}$ In a study by 
Correlation Between Clinical Oral Dryness Score and Salivary Flow Rates in Active and Passive Smokers

Table 1: Descriptive Characteristics of Study Population

\begin{tabular}{|l|c|c|}
\hline Quantitative variables & Mean & SD \\
\hline Age in years & 32.86 & 6.304 \\
\hline Qualitative variables & n & $\%$ \\
\hline Gender & & \\
\hline Female & 72 & 33.2 \\
\hline Male & 145 & 66.8 \\
\hline Ethnicity & & \\
\hline Sindhi & 43 & 19.8 \\
\hline Balochi & 20 & 9.2 \\
\hline Pathan & 15 & 6.9 \\
\hline Punjabi & 12 & 5.5 \\
\hline Urdu & 127 & 58.5 \\
\hline Smokers & & \\
\hline Active & 54 & 24.9 \\
\hline Passive & 163 & 75.1 \\
\hline Betel quid (Pan) & & \\
\hline Yes & 36 & 16.6 \\
\hline No & 181 & 83.4 \\
\hline Areca nut (Chalia) & & \\
\hline Yes & 36 & 16.6 \\
\hline No & 181 & 83.4 \\
\hline & \\
\hline
\end{tabular}

Figure 1: Descriptive Statistics of Salivary Flow Rate

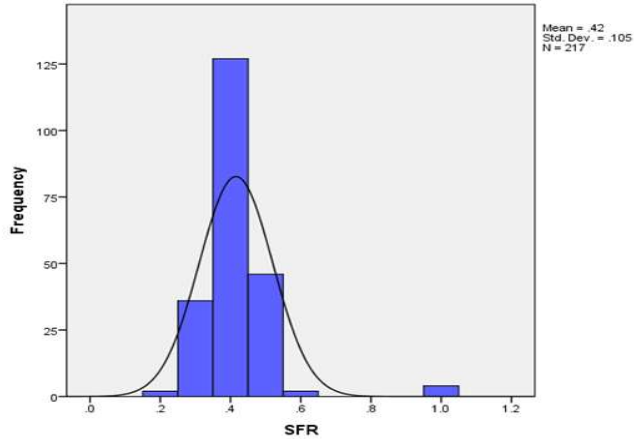

Figure 2: Descriptive Statistics of Clinical Oral Dryness Score

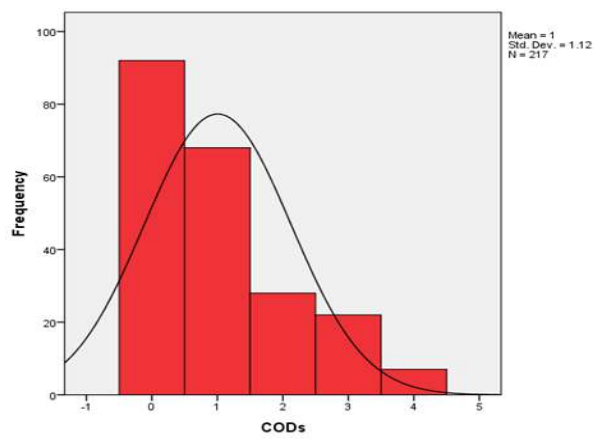

Farsi, it was seen that participants who complained of mouth dryness had significantly lower salivary flow rate as compared to non-complainers. ${ }^{4}$ Osailan et al. also found negative correlation between CODS and $\operatorname{SFR}(p<0.01)$ in their research. ${ }^{3}$ In the study by Jager et al., the relationship between
Table 2: Stratification of Cod Score And Salivary Flow Rate

\begin{tabular}{|c|c|c|c|c|c|c|}
\hline & \multicolumn{3}{|c|}{ CODs } & \multicolumn{3}{|c|}{ SFR } \\
\hline Variables & Mean & SD & P-value & Mean & SD & P-value \\
\hline \multicolumn{7}{|l|}{ Age groups } \\
\hline$</=35$ years & 0.87 & 0.99 & \multirow{2}{*}{0.001} & 0.42 & 0.10 & \multirow{2}{*}{0.01} \\
\hline$>35$ years & 2.59 & 1.27 & & 0.35 & 0.07 & \\
\hline \multicolumn{7}{|l|}{ Gender } \\
\hline Male & 1.14 & 1.11 & \multirow{2}{*}{0.009} & 0.38 & 0.06 & \multirow{2}{*}{0.001} \\
\hline Female & 0.72 & 1.07 & & 0.46 & 0.14 & \\
\hline \multicolumn{7}{|l|}{ Ethnicity } \\
\hline Sindhi & 1.00 & 1.17 & \multirow{5}{*}{0.509} & 0.41 & 0.10 & \multirow{5}{*}{0.631} \\
\hline Balochi & 0.80 & 0.76 & & 0.44 & 0.14 & \\
\hline Pathan & 0.60 & 0.82 & & 0.40 & 0.08 & \\
\hline Punjabi & 1.17 & 1.03 & & 0.39 & 0.05 & \\
\hline Urdu & 1.07 & 1.18 & & 0.41 & 0.10 & \\
\hline \multicolumn{7}{|l|}{ Smokers } \\
\hline Passive & 0.87 & 1.05 & \multirow{2}{*}{0.001} & 0.43 & 0.09 & \multirow{2}{*}{0.001} \\
\hline Active & 1.43 & 1.22 & & 0.37 & 0.14 & \\
\hline \multicolumn{7}{|l|}{ Betel Quid } \\
\hline No & 0.92 & 1.07 & \multirow{2}{*}{0.01} & 0.42 & 0.11 & \multirow{2}{*}{0.001} \\
\hline Yes & 1.44 & 1.22 & & 0.35 & 0.08 & \\
\hline \multicolumn{7}{|l|}{ Areca nut } \\
\hline No & 0.88 & 1.04 & \multirow{2}{*}{0.001} & 0.43 & 0.11 & \multirow{2}{*}{0.001} \\
\hline Yes & 1.61 & 1.29 & & 0.34 & 0.06 & \\
\hline
\end{tabular}

CODS and SFR was evaluated and a weak negative correlation between CODS and hyposalivation group ( $\mathrm{r}=-$ $0.33, \mathrm{p}<0.01$ ), moderate correlation between CODS and normal \& high salivation groups was observed respectively $(\mathrm{r}=-0.56, \mathrm{p}<0.01 \& r=-0.55, \mathrm{p}<0.01) .{ }^{14}$ Hence CODS, being a sensitive tool, can be utilized in general oral examination and for distinguishing patients with normal salivation from those with hyposalivation.

In the current study, the average age of the participants was estimated as 32.86 years. Majority of our selected patients were males $(66.8 \%)$ and less than and equal to thirty five years of age $(92.1 \%)$. Mouth dryness significantly increased with increase in age $(\mathrm{p}<0.05)$ whereas SFR significantly decreased $(\mathrm{p}<0.05)$. Furthermore, males showed significantly high values of CODS and lower values of SFR as compared to females $(\mathrm{p}<0.05)$. Dissimilar findings were witnessed in the study by Farsi in which no statistically significant difference was found in dry oral cavity with respect to age and gender $(\mathrm{p}>0.05) .{ }^{4}$ Ship et al. and Osterberg et al. also showed no change in SFR with increase in age but Nederfors et al. found higher prevalence of oral dryness with increase in age. ${ }^{16-18}$ This might be due to increase in medication intake among elderly patients.

In the present study, the mean SFR of active smokers, betel quid and areca nut consumers was significantly low whereas CODS wassignificantly high as compared to passive smokers 
and non-consumers of betel quid and areca nut $(\mathrm{p}<0.05)$. In the studies by Alaee et al., Singh et al. and Rad M et al. significantly low mean SFR was reported in smokers as compared to non-smokers $(\mathrm{p}<0.05) .{ }^{13,19,20}$ Whereas, the studies by Khan GJ et al. and Rehan et al. found no statistically significant relationship between smokers and SFR $(\mathrm{p}>0.05){ }^{8,21}$ Furthermore Rehan et al. also concluded in their study that tobacco consumption had no effect on resting mouth SFR . Patil et al. in their study showed that consumers of cigarettes or chewable tobacco or both had significant effect on progression of oral abrasions. ${ }^{22}$ In the study by Abdul et al. significantly high ratio of SFR among areca nut consumers (35.7 mm) was reported when compared to SFR of healthy individuals at $3^{\text {rd }} \mathrm{min}(30.7 \mathrm{~mm})$, but with increasing frequency, duration and exposure of areca nut consumption the SFR significantly decreased. ${ }^{23}$ The personal habits such as smoking, consumption of areca nut and betel quid influence the individual's oral health in the long term. Previously, it has been demonstrated that the presence of nicotine in the smoke and alkaloids in areca nut directly affect the SFR as well as oral and dental health. ${ }^{24,25}$ Initially with the consumption of these products the flow of saliva improves, but salivary secretion is usually decreased with long-term use. Among the limitation, it was the single centered study and there was an equal distribution of active and passive smokers, which may affect the correlation. It is recommended that COD score is a useful, simple and adequate tool for the routine investigation for evaluating patients complaining of mouth dryness.

CONCLUSION: Salivary flow rate was significantly decreased with increase in COD score thus having an implication on oral dryness feeling in these participants.

\section{REFERENCES:}

1. Dodds M, Roland S, Edgar M, Thornhill M. Saliva A review of its role in maintaining oral health and preventing dental disease. Bdj Team. 2015;2:15123.

2. Tiwari M. Science behind human saliva. J Nat Sci Biol Med. 2011;2(1):53-8.

3. Osailan SM, Pramanik R, Shirlaw P, Proctor GB, Challacombe SJ. Clinical assessment of oral dryness: development of a scoring system related to salivary flow and mucosal wetness. Oral Surg Oral Med Oral Pathol Oral Radiol. 2012;114(5):597603.

4. Farsi NM. Signs of oral dryness in relation to salivary flow rate, $\mathrm{pH}$, buffering capacity and dry mouth complaints. BMC Oral Health. 2007;7:15.

5. Löfgren CD, Wickström C, Sonesson M, Lagunas PT, Christersson C. A systematic review of methods to diagnose oral dryness and salivary gland function. BMC Oral Health. 2012;12(1):29.

6. Wolff MS, Kleinberg I. The effect of ammonium glycopyrrolate (Robinul)-induced xerostomia on oral mucosal wetness and flow of gingival crevicular fluid in humans. Arch Oral Biol. 1999;44(2):97-102.

7. Shimazaki Y, Fu B, Yonemoto K, Akifusa S, Shibata Y, Takeshita T, et al. Stimulated salivary flow rate and oral health status. J Oral Sci. 2017;59(1):55-62.
8. Rehan F, Khan BRS, Memon MS, Naqvi S, Khan R, Sultan $\mathrm{Z}$. Analysis of resting mouth salivary flow rate and salivary $\mathrm{pH}$ of tobacco chewers and smokers. J Pak Dent Assoc. 2016;4:159-63.

9. Challacombe SJ, Proctor GB. Clinical assessment. Bdj. 2014;217:486.

10. Hijjaw O, Alawneh M, Ojjoh K, Abuasbeh H, Alkilany A, Qasem N, et al. Correlation between Xerostomia index, Clinical Oral Dryness Scale, and ESSPRI with different hyposalivation tests. Open Access Rheumatol. 2019;11:118.

11. Sreebny LM, Valdini A, Yu A. Xerostomia. Part II: Relationship to nonoral symptoms, drugs, and diseases. Oral Surg Oral Med Oral Pathol. 1989;68(4):419-27.

12. Ship JA, Fox PC, Baum BJ. How much saliva is enough? 'Normal' function defined. J Am Dent Assoc. 1991;122(3):639.

13. Rad M, Kakoie S, Niliye Brojeni F, Pourdamghan N. Effect of Long-term Smoking on Whole-mouth Salivary Flow Rate and Oral Health. J Dent Res Dent Clin Dent Prospects. 2010;4(4):110-4.

14. Jager DHJ, Bots CP, Forouzanfar T, Brand HS. Clinical oral dryness score: evaluation of a new screening method for oral dryness. Odontol. 2018;106(4):439-44.

15. Correia J, Martins A, Romao V, Pinto J, Gonçalves A, Simão R, et al. Association Of Clinical Oral Dryness Score With Unstimulated Salivary Flow Rate In Xerostomia Patients. Med Oral Patol Oral Cir Bucal2017.

16. Ship JA, Baum BJ. Is reduced salivary flow normal in old people? Lancet. 1990;336(8729):1507.

17. Osterberg T, Birkhed D, Johansson C, Svanborg A. Longitudinal study of stimulated whole saliva in an elderly population. Scand J Dent Res. 1992;100(6):340-5.

18. Nederfors T, Isaksson R, Mornstad H, Dahlof C. Prevalence of perceived symptoms of dry mouth in an adult Swedish population--relation to age, sex and pharmacotherapy. Community Dent Oral Epidemiol. 1997;25(3):211-6.

19. Singh P. Collaborating centres: Rediscovering an extended arm of World Health Organization. J Indian Assoc Public Health Dent. 2015;13(1):11-3.

20. Alaee A,Azizi A, Valaei N, Moeini SH. The Correlation between Cigarette Smoking and Salivary Flow Rate J Res Dentomaxillofac Sci. 2017;2(3):5-9.

21. Khan GJ, Javed M, Ishaq M. Effect of smoking on salivary flow rate. Gomal Journal of Medical Sciences. 2010;8(2).

22. Patil PB, Bathi R, Chaudhari S. Prevalence of oral mucosal lesions in dental patients with tobacco smoking, chewing, and mixed habits: A cross-sectional study in South India. J Family Community Med. 2013;20(2):130-5.

23. Abdul Khader NF, Dyasanoor S. Assessment of Salivary Flow Rate and $\mathrm{pH}$ Among Areca Nut Chewers and Oral Submucous Fibrosis Subjects: A Comparative Study. J Cancer Prev. 2015;20(3):208-15.

24. Petrusic N, Posavac M, Sabol I, Mravak-Stipetic M. The Effect of Tobacco Smoking on Salivation. Acta Stomatol Croat. 2015;49(4):309-15.

25. Venkatesh D, Puranik RS, Vanaki SS, Puranik SR. Study of salivary arecoline in areca nut chewers. J Oral Maxillofac Pathol. 2018;22(3):446. 